\title{
Um Sistema Web Gamificado para a Aprendizagem de Lógica Formal
}

\author{
Jefferson O. Andrade ${ }^{1}$, Marta Canese $^{2}$ \\ ${ }^{1}$ Instituto Federal de Educação, Ciência e Tecnologia do Espírito Santo (IFES) \\ Coordenação de Informática - Campus Serra \\ Rodovia Es-010 - Km 6,5 - Manguinhos - 29173-087 - Serra - ES - Brazil \\ ${ }^{2}$ Facultad de Postgrado - Universidad del Norte \\ Brasil 184 e/ Mcal. López y José Berges - Asunción - Paraguay \\ joandradedifes.edu.br, mcanese@gmail.com
}

\begin{abstract}
Formal logic plays a fundamental role in computer science. Unfortunately, it is observed that the effectiveness of the learning process of formal logic has proved to be less than ideal. This work presents the $\exists$ lementar system; a system that integrates a proof assistant in a gamified context, i.e., that uses game elements to promote student's engagement and motivation. After introducing $\exists$ lementar's main features, this work presents preliminary data that point to an improvement in student's performance indicators.
\end{abstract}

Resumo. A lógica formal possui um papel fundamental na ciência da computação. Infelizmente, se observa que a eficácia do processo de ensinoaprendizagem da lógica formal tem se mostrado abaixo do ideal. Este trabalho apresenta o sistema ヨlementar; um sistema que incorpora um assistente de provas em um contexto de gamificação, i.e., que utiliza elementos de jogos para promover o engajamento e a motivação dos alunos. Após introduzir as características gerais do sistema $\exists$ lementar, este artigo apresenta dados preliminares que indicam melhoria nos indicadores de desempenho dos alunos.

\section{Introdução}

O domínio dos conceitos de lógica formal é uma competência fundamental para o profissional de computação. A lógica desempenha um papel tão fundamental na ciência da computação quanto o cálculo diferencial e integral desempenha nas engenharias tradicionais. Infelizmente, observa-se que a eficiência do processo de ensino-aprendizagem da lógica matemática nos cursos de computação, de modo geral, tem se mostrado abaixo do ideal, fazendo com que haja um grande número de reprovações na disciplina [Setti 2009].

Evidentemente, esta situação é indesejável. Assim, visando aumentar a efetividade do processo de ensino-aprendizagem da lógica formal, foi formulada uma proposta de intervenção didática através do desenvolvimento e utilização de um software educacional que mescle dois conceitos distintos, descritos abaixo.

Primeiramente, o software educacional proposto deveria implementar um assistente de provas para uso educacional. Boa parte da dificuldade encontrada pelos alunos na aprendizagem da lógica formal se encontra na construção de provas formais. 
O uso de assistentes de prova tem se mostrado útil para ultrapassar esta dificuldade [Wiedijk and van Raamsdonk 2007, Kameyama and Sato 2006].

Em segundo lugar, para estimular a motivação dos alunos, o software proposto deveria empregar elementos tipicamente associados aos videogames, porém em um contexto diferente do contexto do mero entretenimento, ou seja, propomos desenvolver um software educacional que atuasse como apoio pedagógico à aprendizagem da lógica formal, mas que empregue elementos típicos de jogos em um contexto extra-jogos. Esta abordagem vem sendo chamada de "gamificação" [Deterding et al. 2011].

Para concretizar a intervenção pedagógica proposta, foi realizado um estudo que fundamentou o projeto de um sistema com as características de gamificação desejadas [Andrade and Canese 2013], este sistema foi denominado $\exists$ lementar, e um protótipo foi implementado e posto em uso. O sistema $\exists$ lementar, incorpora como backend o sistema $\forall$ DeL, que é um assistente de provas para uso educacional desenvolvido anteriormente pelo primeiro autor deste artigo. Neste artigo exploraremos principalmente as características associadas com o uso de gamificação do $\exists$ lementar.

\section{Metodologia}

O protótipo desenvolvido para o sistema $\exists$ lementar teve como objetivo principal a experimentação com os conceitos de gamificação. Neste sentido, foram feitas escolhas com relação a que características de gamificação - de acordo com a taxonomia proposta por [Petrović and Ivetić 2012] - poderiam ser implementadas, dadas as restrições de pessoal e tempo para a implementação do protótipo. Ao todo foram escritas mais de 10500 linhas de código para o sistema $\forall$ DeL, e mais de 3500 linhas de código para os sistema $\exists$ lementar.

Algumas estratégias foram adotadas para atingir melhor interação com os alunos. Uma delas foi a de apresentar dicas sobre o uso de regras de dedução para servir de apoio aos estudantes, bem como utilizar a estratégia de revelação progressiva das informações. Além disso, o sistema é totalmente interativo (ao invés de operar em batch como a maioria dos assistentes de prova), de modo que, a cada regra aplicada pelo aluno, o estado da prova é atualizado e exibido, oferecendo feedback imediato das ações executadas. Caso o aluno tente aplicar uma regra de modo incorreto, o sistema exibe uma mensagem explicando o motivo do erro e, em certos casos, sugerindo correções.

Do ponto de vista das interações sociais, se considerou necessário que o sistema atuasse em duas dimensões distintas: (a) atuar como veículo, possibilitando as interações sociais entre seus usuários; (b) atuar de modo a suscitar nos usuários o sentimento de obrigação social, ou seja, despertar nos usuários o sentimento de necessidade de interagir com o sistema de modo a se sentir parte de um círculo social. A primeira destas dimensões, a da interação social foi trabalhada, através das interações presenciais, em sala de aulas e no laboratório, e também através do estímulo à participação dos alunos em fóruns de discussão. A segunda dimensão, a de obrigação social, foi estimulada através do uso de mecanismos de competição tais como rankings, conquista de distintivos e por notificações sobre o progresso dos "amigos" do aluno no sistema.

Incorporar a dimensão de competição é bastante importante no uso de gamificação e essa importância se refletiu no design do sistema $\exists$ lementar em vários pontos. Para que 
se pudesse estabelecer um sistema de competição, foi necessário introduzir a ideia de pontuação associada à resolução dos problemas; esta pontuação é instrumental tanto para o critério de autocompetição quanto para a competição externa (com outros usuários). $\mathrm{O}$ sistema também permite outras formas de competição, como por exemplo, a competição informal entre os usuários pela comparação do número e do tipo de distintivos obtido por cada um. Os distintivos permitem que o usuário receba (e exiba) reconhecimento por uma ampla variedade de realizações, tais como ser o primeiro a resolver um determinado exercício, resolver 50 exercícios em sequência, resolver exercícios todos os dias por 10 dias seguidos, e assim por diante.

Talvez tão importante quanto a dimensão de competição para os sistemas gamificados, seja a dimensão de mecânica de jogos, ou seja, a forma como os usuários interagem com o sistema. Em alguns domínios de aplicação, esta mecânica pode lembrar um videogame, em outros, devido a natureza do domínio, haverá restrições na implantação de uma mecânica lúdica. O sistema $\exists$ lementar não se parece com um videogame. A criação de provas no sistema de dedução natural não de adapta a este tipo de mecânica. Entretanto, foi feito um esforço para aproximar a experiência de uso do sistema o máximo possível de um design jogável. $\mathrm{O} \exists$ lementar permite criar provas no sistemas de dedução natural, no estilo Fitch [Barwise and Etchemendy 1999], tanto para argumentos formais na lógica proposicional quanto para a lógica de predicados. A sintaxe utilizada para gerar os passos das provas é, em essência, a transcrição daquilo que o estudante escreveria se estivesse realizando a prova "em papel", com a diferença de que o estudante apenas precisa indicar a regra que será empregada e seus argumentos, à partir disto o sistema calcula a nova fórmula a ser acrescentada à prova.

O sistema $\exists$ lementar também incorpora funcionalidades voltadas ao acompanhamento do progresso dos alunos por parte do professor. Estes requisitos incluem a possibilidade de visualizar as soluções geradas pelos alunos, e a possibilidade de consultar as estatísticas relacionadas a cada aluno, tais como frequência com que o aluno acessa o sistema, pontuação, listagem de problemas resolvidos e distintivos obtidos.

\section{O Sistema $\exists$ lementar}

O sistema $\exists$ lementar foi implementado como uma aplicação web. A página inicial do sistema Đlementar exibe um layout minimalista onde é possível ver uma mensagem de boas-vindas na região central da página e um menu com algumas operações básicas na região esquerda, chamada de área de navegação.

A Figura 1 apresenta um excerto da página de listagem de listagem de problemas, conforme exibida para um usuário com perfil de administrador. Os problemas são exibidos de modo "paginado". Nesta página o link 'Provar' leva à página que dá acesso à interface do assistente de provas propriamente dito. Nesta página será possível ao estudante tentar solucionar o problema. Se o usuário possuir status de administrador do sistema, o link 'Editar' estará visível e permitirá alterar as informações relacionadas ao problema, incluindo o enunciado formal do mesmo (argumento).

A página de progresso tem por objetivo exibir uma representação visual das conquistas do estudante. Nesta página são exibidos os problemas já resolvidos pelo estudante assim como os troféus já conquistados. A Figura 2 mostra a página de progresso de um usuário. Na primeira parte da página, sob o título 'Problemas Resolvidos' é mostrada 


\section{Lista de Problemas}

\begin{tabular}{|c|c|c|c|c|c|c|}
\hline \# & Tipo & Argumento & Criado em & Resolvido por & & \\
\hline 5 & Propositional & $A \vdash A v B$ & $2013 / 03 / 20$ & 2 & Provar & Editar \\
\hline 6 & Propositional & $A ; B \vdash A \wedge B$ & $2013 / 03 / 20$ & 1 & Provar & Editar \\
\hline 7 & Propositional & $B \vdash A v B$ & $2013 / 03 / 20$ & 0 & Provar & $\underline{\text { Editar }}$ \\
\hline 8 & Propositional & $(\mathrm{A} \wedge \mathrm{B}) \rightarrow \mathrm{C} \vdash \mathrm{A} \rightarrow(\mathrm{B} \rightarrow \mathrm{C})$ & $2013 / 03 / 20$ & 1 & Provar & $\underline{\text { Editar }}$ \\
\hline 9 & Propositional & $\neg \mathrm{A} \vee \mathrm{B} \vdash \mathrm{A} \rightarrow \mathrm{B}$ & $2013 / 03 / 20$ & 1 & Provar & Editar \\
\hline 10 & Propositional & $\mathrm{A} \rightarrow \mathrm{B} ; \mathrm{B} \rightarrow \mathrm{C} \vdash \mathrm{A} \rightarrow \mathrm{C}$ & $2013 / 03 / 21$ & 2 & Provar & Editar \\
\hline 11 & Propositional & $A \vee B ; A \rightarrow C ; B \rightarrow C \vdash C$ & $2013 / 03 / 27$ & 1 & Provar & Editar \\
\hline 12 & Propositional & $A v B ; \neg A \vdash B$ & $2013 / 03 / 27$ & 1 & Provar & Editar \\
\hline
\end{tabular}

Figura 1. Página de listagem de problemas do protótipo do $\exists$ lementar em uso por um usuário administrador.

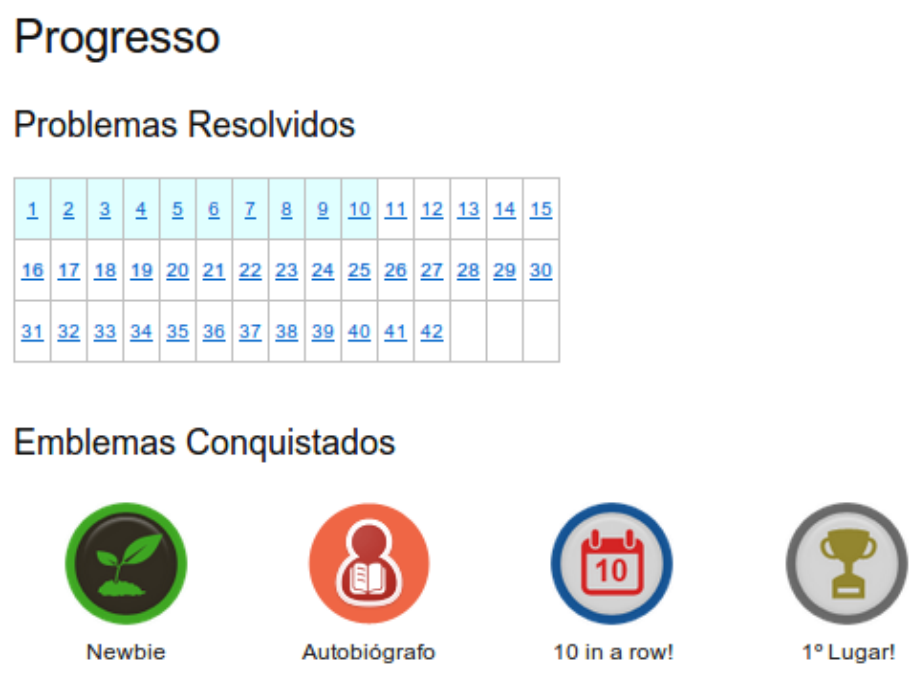

Figura 2. Página de progresso exibida pelo protótipo do $\exists$ lementar.

uma tabela com todos os problemas disponíveis no sistema; os problema que o usuário resolveu estão destacados (problemas de 1 a 10 neste exemplo) e é possível, através de um link, ter acesso direto ao enunciado dos problemas. Na segunda parte da página de progresso, sob o título 'Emblemas Conquistados' aparecem os distintivos que o usuário conquistou até o momento. Os distintivos são mostrados através de sua representação gráfica e do nome do distintivo.

A página de provas é a interface entre os estudantes e o assistente de provas de fato, ou seja, esta página encapsula as funcionalidades do sistema $\forall$ DeL e permite aos estudantes acessarem estas funcionalidades através da aplicação web do sistema $\exists$ lementar. Espera-se que esta página seja o recurso do protótipo que os estudantes mais acessem e gastem mais tempo utilizando.

A Figura 3 apresenta o assistente de provas imediatamente após a prova ter sido concluída com sucesso. Pode-se verificar a presença de diversas fórmulas geradas pela aplicação de regras de dedução, associadas a comandos emitidos pelo usuário. Pode-se 


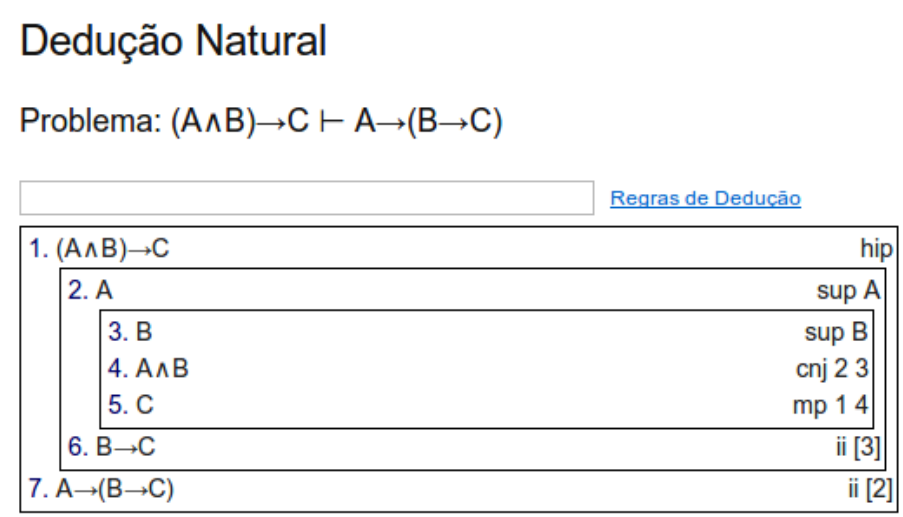

\section{Q.E.D}

Figura 3. Página do assistente de provas do protótipo do $\exists$ lementar.

\begin{tabular}{|c||}
\hline \multicolumn{1}{|c||}{ Atenção! } \\
\begin{tabular}{|c||} 
A regra Modus Ponens $(m p)$ nāo pode ser aplicada às fórmulas 4 \\
$(A \wedge B)$ e 2 (A). Os argumentos desta regras devem se compativeis \\
com os padrōes " $\alpha$ " $\mathrm{e}$ " $\alpha \rightarrow \beta$ ".
\end{tabular} \\
\hline \hline [Fechar] \\
\hline \hline
\end{tabular}

Figura 4. Mensagem de erro emitida pelo sistema $\exists$ lementar em resposta a um comando impróprio do usuário.

verificar também, que nesta prova em particular utilizou-se duas sub-provas, que estão corretamente representadas graficamente pelo uso de retângulos englobando os diversos níveis de provas. O término com sucesso da prova é sinalizado pelo uso do marcador "Q.E.D". ${ }^{1}$

No topo da página de prova é apresentado o enunciado formal do problema que se pretende resolver, abaixo do título 'Dedução Natural' e à frente do rótulo 'Problema:'. Ao ser ativada, a página de prova inicia compulsoriamente uma nova prova introduzindo-se as hipóteses do problema como as primeiras fórmulas do sequente. À partir deste ponto, o usuário deve emitir comandos que instruam o assistente de provas sobre quais regras devem ser aplicadas, e quais os argumentos que estas regras receberão. Por exemplo, para iniciar uma nova sub-prova à partir da suposição de que a fórmula $A$ é verdadeira, $o$ usuário deve emitir o comando "sup A". Para aplicar a regra de modus ponens, tendo as fórmulas de índice 1 e 4 como argumento, o usuário deve emitir o comando "mp 14 ".

Caso ocorra algum erro na execução do comando, o sistema emitirá uma mensagem de erro esclarecendo o problema e tentando indicar como o mesmo pode ser solucionado. A Figura 4 mostra uma mensagem de erro emitida em resposta à uma tentativa do usuário de aplicar a regra de modus ponens às fórmulas 2 e 4 do exemplo mostrado na Figura 3.

Caso o usuário queira relembrar quais são as regras de dedução disponíveis no sistema, o link "Regras de Dedução" exibe uma página com a descrição de todas as regras de dedução conhecidas do sistema. Uma vez que há regras de dedução de diferentes naturezas, a página de informações agrupa as regras em duas categorias: regras de equivalência

\footnotetext{
${ }^{1}$ Do latim Quod Erat Demonstrandum, "como se queria demonstrar".
} 
e regras de inferência.

A Figura 5 mostra um excerto da página de informações sobre regras. Nesta figura temos um excerto da listagem de regras de equivalência, porém o sistema também gera a listagem das regras de inferências. Ambas as listagens são geradas automaticamente à partir das definições das regras de dedução, de modo que, se uma nova regra de dedução for inserida no sistema, não será necessário alterar a página de informações.

\begin{tabular}{|c|c|c|}
\hline Abrev. & Nome & Definiçāo \\
\hline dn & DoubleNegation & $\neg \neg \alpha \equiv \alpha$ \\
\hline bicond & Biconditional & $\alpha \leftrightarrow \beta \equiv \alpha \rightarrow \beta \wedge \beta \rightarrow \alpha$ \\
\hline $\mathrm{cmtc}$ & CommutativityCnj & $\alpha \wedge \beta \equiv \beta \wedge \alpha$ \\
\hline cmtd & CommutativityDsj & $\alpha \vee \beta \equiv \beta v \alpha$ \\
\hline assc & AssociativityCnj & $a v \beta v Y \equiv \alpha v \beta v Y$ \\
\hline assd & AssociativityDsj & $\alpha \wedge \beta \wedge \gamma \equiv \alpha \wedge \beta \wedge \gamma$ \\
\hline dst1 & Distributivity1 & $\alpha \wedge \beta v \gamma \equiv(\alpha \wedge \beta) v(\alpha \wedge \gamma)$ \\
\hline dst2 & Distributivity2 & $\alpha v(\beta \wedge Y) \equiv \alpha v \beta \wedge \alpha v Y$ \\
\hline
\end{tabular}

Figura 5. Excertos da página de descrição das Regras de Dedução exibida pelo $\exists$ lementar.

O sistema $\exists$ lementar permite a definição das regras de dedução através de uma "linguagem específica de domínio" (Domain Specific Language - DSL) desenvolvida especialmente para esta finalidade como parte do sistema $\forall$ DeL. A linguagem desenvolvida para o $\forall$ DeL permite tanto a definição de regras de equivalência quanto a definição de regras de inferência.

Também cabe ressaltar que, embora o sistema não possuía uma interface específica para dispositivos móveis, interface web do sistema $\exists$ lementar foi construída para poder ser utilizada mesmo em dispositivos com telas relativamente reduzidas e utiliza apenas tecnologias web abertas e padronizadas, o que permite sua utilização em uma ampla gama de dispositivos móveis.

\section{Experimento e Resultados}

Foi feito um experimento com o uso do protótipo do sistema $\exists$ lementar em uma turma da disciplina de Lógica do curso de Bacharelado em Sistemas de Informação do Campus Serra do Instituto Federal do Espírito santo (IFES), no período 2012-2. O experimento em questão se constituiu em introduzir o uso de gamificação, com o apoio do protótipo do sistema Đlementar, na condução de uma instância da disciplina de Lógica no período 2012-2. Para a análise quantitativa da efetividade da introdução de gamificação no processo de ensino-aprendizagem da lógica matemática foram levantados diversos indicadores, alguns dos quais são mostrados na Tabela 1. Esta tabela apresenta um resumo dos dados relevantes referentes aos indicadores de notas obtidos pelos alunos na disciplina de Lógica ao longo dos 6 períodos analisados neste estudo (de 2010/1 a 2012/2).

Como pode ser observado na coluna Alunos da Tabela 1, o número de alunos matriculados na disciplina de Lógica gira em torno de 55 por período letivo. O acentuado decréscimo observado no período 2011/2 se deveu ao fato de que neste semestre não 
Tabela 1. Indicadores estatísticos levantados para a disciplina de Lógica.

\begin{tabular}{|c|c|c|c|c|c|c|c|}
\hline Período & Alunos & Não Des. & Des. & $\mathbf{E}\left(\mathbf{L}_{\mathbf{1}}\right)$ & $\sigma\left(\mathbf{L}_{\mathbf{1}}\right)$ & $\mathbf{E}\left(\mathbf{L}_{\mathbf{2}}\right)$ & $\sigma\left(\mathbf{L}_{\mathbf{2}}\right)$ \\
\hline \hline $2010 / 1$ & 54 & 39 & 15 & 36,2 & 22,7 & 45,1 & 23,3 \\
\hline $2010 / 2$ & 57 & 52 & 5 & 59,2 & 18,4 & 52,6 & 17,5 \\
\hline $2011 / 1$ & 56 & 56 & 0 & 51,1 & 19,7 & 38,7 & 16,0 \\
\hline $2011 / 2$ & 26 & 26 & 0 & 64,3 & 19,2 & 44,8 & 25,1 \\
\hline $2012 / 1$ & 53 & 35 & 18 & 49,9 & 18,2 & 39,1 & 27,4 \\
\hline $2012 / 2$ & 54 & 46 & 8 & 55,2 & 21,0 & 58,4 & 17,8 \\
\hline
\end{tabular}

houve ingresso de turma nova no curso. Deste modo, cursaram a disciplina neste período apenas alunos repetentes ou alunos que optaram por não cursar a disciplina quando ingressaram no curso.

Também é possível observar nas colunas Não Des. e Des. da Tabela 1, a quantidade de alunos não-desistentes e desistentes ${ }^{2}$, respectivamente, na disciplina de Lógica em cada um dos seis períodos analisados neste estudo. Esperava-se que o recesso acadêmico no período 2012/2 contribuísse para um alto índice de não-conclusão da disciplina, entretanto, o índice de conclusão para o período de $2012 / 2$ foi de $62.9 \%$, o que representa um aumento de mais de $50 \%$ em relação ao período anterior. Este período teve início em novembro de 2012 e término abril de 2013, com um período de recesso de 21 dias no mês de janeiro de 2013. Nota-se que foi um período atípico, devido à adequação do calendário acadêmico a duas greves prolongadas ocorridas nos anos de 2011 e 2012 na maioria das instituições federais de ensino superior do Brasil, incluindo o IFES.

É importante reiterar que não se pode presumir que o uso de gamificação e de um assistente de provas foi o único responsável por esta melhora no índice de não desistência, mas este é certamente um indicador importante a ser considerado.

Como já foi dito, a disciplina de Lógica trata também de outros conteúdos periféricos à lógica formal, porém, tais conteúdos não foram abordados na proposta de gamificação do aprendizado de lógica elaborada neste estudo, assim, para tentar analisar e entender melhor o impacto desta proposta, considerou-se que uma apresentação e análise das avaliações específicas de lógica formal seria útil. Tipicamente a disciplina de lógica é avaliada por meio de 4 instrumentos: três avaliações teóricas e um trabalho prático. Destas três avaliações teóricas, duas se focam especificamente na lógica formal. Estas duas avaliações com foco em lógica formal são as avaliações denominadas $L_{1}$ e $L_{2}$ na Tabela 1.

As quatro últimas colunas da Tabela 1 representam: $\mathbf{E}\left(\mathbf{L}_{1}\right)$ - média das notas obtidas na primeira prova de lógica formal; $\sigma\left(\mathbf{L}_{\mathbf{1}}\right)$ - desvio padrão das notas obtidas na primeira prova de lógica formal; $\mathbf{E}\left(\mathbf{L}_{2}\right)$ - média das notas obtidas na segunda prova de lógica formal; $\sigma\left(\mathbf{L}_{\mathbf{2}}\right)$ - desvio padrão das notas obtidas na segunda prova de lógica formal. Para se computar as médias e desvios padrões das notas foram considerados apenas os alunos não-desistentes.

Se for considerado especificamente o período de utilização do protótipo de sistema $\exists$ lementar pelos alunos da disciplina de lógica, veremos que o maior impacto do uso deste

\footnotetext{
${ }^{2}$ São considerados "desistentes" os alunos que frequentaram menos do que $25 \%$ da aulas.
} 
sistema ocorreu na segunda prova de lógica formal $\left(E\left(L_{2}\right)\right)$, pois esta ocorreu algumas semanas após a disponibilização do sistema para uso dos alunos. Antes disto, embora se tenha procurado manter os alunos de algum modo envolvidos com o desenvolvimento do sistema, não havia utilização real ou efeito concreto do uso de gamificação.

Se for analisada a evolução histórica da média das notas da segunda avaliação de lógica formal até o período de 2012/1, conforme mostrado na Figura 6(a), será possível notar uma tendência de declínio destas notas, conforme fica evidenciado pela aproximação linear mostrada naquela figura. Embora haja alguma oscilação nos valores, a tendência descendente é clara. Por outro lado, ao se considerar também a média da nota da segunda avaliação de lógica formal para o período de 2012/2, conforme mostrado na Figura 6(b), verifica-se não apenas que esta média é significativamente superior a todas às outras médias registradas, mas também que a inclusão desta informação foi suficiente para reverter a tendência estatística de declínio nas notas, como evidenciado pela aproximação linear mostrada naquela figura.

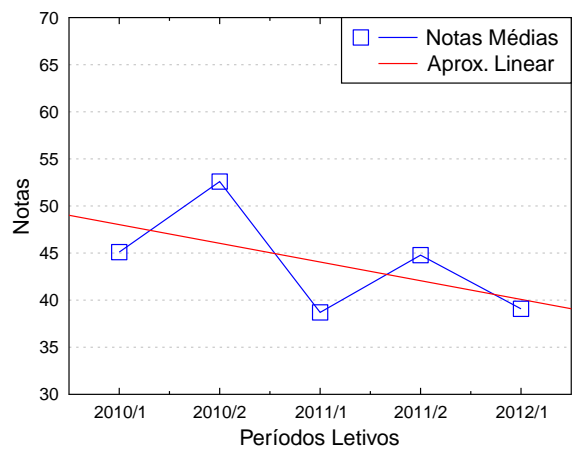

(a) Médias das notas da $2^{\circ}$ prova até $2012 / 1$.

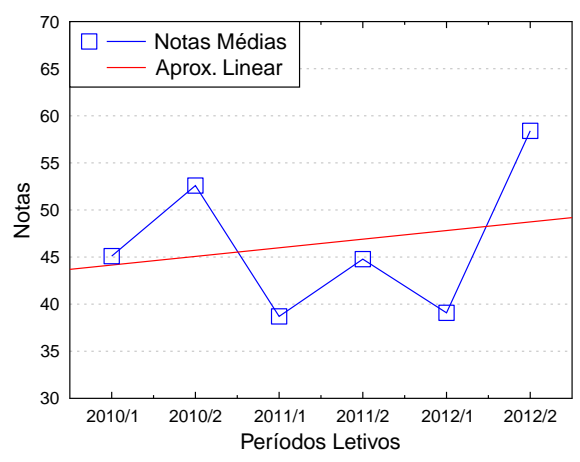

(b) Médias das notas da $2^{\circ}$ prova até 2012/2.

Figura 6. Médias das notas da $2^{\mathrm{a}}$ prova de lógica formal.

Deve ser notado que a média da segunda avaliação de lógica formal para o período 2012/2 representa não apenas um aumento de mais de $49 \%$ em relação ao período anterior, mas também representa um aumento de mais de $11 \%$ em relação à maior média já registrada para esta avaliação. Estes indicadores sugerem que houve uma correlação entre o uso do assistente de provas gamificado $\exists$ lementar e a melhoria de performance dos alunos, ao menos na segunda avaliação de lógica formal.

Apesar de alguns contratempos que se interpuseram no desenvolvimento do presente trabalho, consideramos razoável dizer que este estudo obteve resultados animadores. Apesar de todos os prognósticos negativos gerados pela conjuntura social e política delicada em que se viu inserida a educação superior pública brasileira nos últimos dois anos, pode-se verificar melhoria em todos os indicadores analisados para o período de 2012/2, no qual foi realizado o experimento, se comparado com os três períodos anteriores. Além disso, em todos os indicadores analisados, quando não se obteve os melhores resultados já registrados, se obteve resultados muito próximos dos melhores registrados.

\section{Trabalhos Relacionados}

Outros sistemas assistentes ou editores de prova existem para diversos tipos de lógicas. Antes desta pesquisa apontar a necessidade de se desenvolver o sistema $\exists$ lementar, diver- 
sos destes sistemas foram analisados como potenciais candidatos a ferramentas de apoio ao processo de ensino-aprendizagem da lógica formal, e alguns destes merecem destaque.

Sistemas como Coq (http://coq.inria.fr) e HOL (http://hol. sourceforge.net/) são provadores de teorema baseados em lógicas de alta ordem e, embora sejam bastante poderosos como ferramentas de prova, são pouco adequados enquanto ferramentas de apoio à aprendizagem de lógica por alunos iniciantes. Alguns outros sistemas como Otter/Mace2 (http: / / www . cs . unm . edu / mccune / mace 2/) ou seu sucessor, Prover9/Mace4 (http://www. cs.unm. edu/ mccune/ prover 9/) são ferramentas para prova automática de teoremas, o que inviabiliza seu uso como ferramenta de apoio ao aprendizado da construção de provas. Outros sistemas, tais como CAL [Kameyama and Sato 2006], por exemplo, embora tenham sido desenvolvidos como ferramentas de apoio ao aprendizado exigem um conhecimento avançado de programação por parte dos usuários.

Dentre os sistemas estudados, os que mais se adequam ao propósito de atuar como ferramentas de apoio ao aprendizado de lógica formal por alunos iniciantes, são os sistemas na categoria dos editores de provas tais como o JAPE (http://Www . cs.ox.ac.uk/people/bernard.sufrin/personal/jape.org/) e o Pandora (http://www.doc.ic.ac.uk/pandora/). Estes softwares, embora sejam de uso relativamente simples, e sejam eficientes enquanto editores de prova não incluem as características de gamificação que esta pesquisa visava explorar. Esta também é a principal diferença entre o sistema Heráclito [Penteado and Gluz 2011, Mossman et al. 2012] e o sistema $\exists$ lementar descrito neste estudo. Embora tanto o sistema Heráclito quanto o $\exists$ lementar se apresentem ao usuário final como sistema de edição/verificação de provas, o $\exists$ lementar integra este recurso dentro do contexto de um sistema gamificado, buscando trabalhar também as dimensões sociais e de competição, principalmente, junto aos alunos.

\section{Conclusões e Trabalhos Futuros}

No campo do uso de assistentes de prova como ferramentas de ensino, como já citado na seção anterior, esta pesquisa ofereceu não apenas resultados teóricos, como também gerou um "produto" prático e palpável na forma do sistema $\forall$ DeL. Este sistema representa uma contribuição importante para o desenvolvimento tanto da área de assistentes de prova educacionais, quando do ensino de lógica formal de modo geral. Além disso, os potenciais benefícios do sistema $\forall$ DeL, não estão confinados ao desenvolvimento desta pesquisa. $O$ sistema está disponível na forma de um software livre e pode ser acessado por qualquer pessoa interessada. ${ }^{3}$

No tema da gamificação do ensino de lógica formal, foi apresentada uma proposta para a criação de um sistema de ensino de lógica formal que empregue gamificação, e um protótipo do sistema proposto foi implementado e utilizado em caráter experimental. Além da proposta desenvolvida nesta pesquisa, o protótipo do sistema $\exists$ lementar, também representa uma grande contribuição ao estudo da gamificação da educação. Assim como o sistema $\forall$ DeL, o $\exists$ lementar está disponível à comunidade acadêmica, ou a qualquer pessoa interessada, tanto na forma de um sistema web que pode ser utilizado gratuitamente, quanto na forma de software livre através de seu código fonte. ${ }^{4}$

\footnotetext{
${ }^{3}$ http://logica.sr.ifes.edu.br.

${ }^{4}$ http: //logica.sr.edu.br/elementar.
} 
Esta pesquisa fez contribuições, mas também gerou novas indagações e chamou a atenção para outras possíveis linhas de pesquisa. Primeiramente, se apresenta como evidente a necessidade de prosseguir no desenvolvimento do sistema $\exists$ lementar. Devido a restrições de recursos e tempo, o protótipo desenvolvido não incorporou todos os requisitos levantados. O próximo passo lógico na continuidade de nossa pesquisa é, portanto, gerar uma nova versão do protótipo que incorpore os demais requisitos.

Outra possível linha de pesquisa que pode ser explorada é a avaliação do efeito dos estilos de aprendizagem propostos por [Kolb et al. 1984] nos índices de motivação dos estudantes de lógica formal, e também quais as relações entre os diferentes estilos de aprendizagem e o uso de gamificação no ensino.

\section{Referências}

Andrade, J. O. and Canese, M. (2013). ᄏlementar: Aplicando gamificação ao processo de ensino-aprendizagem da lógica formal. In Anais da Oitava Conferência Latinoamericana de Objetos e Tecnologias de Aprendizagem (LACLO 2013), Valdivia Chile.

Barwise, J. and Etchemendy, J. (1999). Language, Proof and Logic. Seven Bridges Press.

Deterding, S., Sicart, M., Nacke, L. E., O'Hara, K., and Nixon, D. (2011). Gamification: Using game design elements in non-gaming contexts. In Proc. of the 2011 Annual Conference on Human factors in Computing Systems - CHI 2011.

Kameyama, Y. and Sato, M. (2006). E-learning of foundation of computer science. In Proc. AEARU Workshop on Network Education (AWNE2006), pages 169-181, Taipei.

Kolb, D. A. et al. (1984). Experiential learning: Experience as the source of learning and development, volume 1. Prentice-Hall Englewood Cliffs, NJ.

Mossman, M., dos S. Gomes, L., and Gluz, J. C. (2012). Objetos de aprendizagem móveis para ensino de dedução natural na lógica proposicional. In Anais do $23^{\circ}$ Simpósio Brasileiro de Informática na Educação (SBIE 2012), page pp. 5, Rio de Janeiro, RJ.

Penteado, F. and Gluz, J. a. C. (2011). Sistema heráclito: Suporte a objetos de aprendizagem interativos e dialéticos voltados ao ensino de dedução natural na lógica proposicional. In Anais do $22^{\circ}$ Simpósio Brasileiro de Informática na Educação (SBIE 2011), pages 244-253, Aracaju, SE.

Petrović, V. and Ivetić, D. (2012). Gamifying education: A proposed taxonomy of satisfaction metrics. In Proc. 8th International Scientific Conference eLearning and software for Education, pages 345-350.

Setti, M. d. O. G. (2009). O Processo de Discretização do Raciocínio Matemático na Tradução para o Raciocínio Computacional. Tese (doutorado), Universidade Federal do Paraná, Curitiba, PR.

Wiedijk, C. K. F. and van Raamsdonk, M. H. F. (2007). Teaching logic using a state-ofthe-art proof assistant. PATE'07, page 33. 